\section{El desarrollo personal de los jóvenes de Medellín, Colombia: más allá de las conductas de riesgo y de resiliencia}

Personal development of youth in Medellín, Colombia: beyond risk behaviors and resilience

\section{Desenvolvimento pessoal entre jovens em Medellín, Colômbia: para além de comportamentos de risco e resiliência}

Dora M. Hernández-Holguín 1 Isabel C. Sánchez 1 Esteban Páez 1

Erika Maria Montoya-Vásquez 1

\title{
Resumen
}

El estudio de los jóvenes se ha ido afianzando hacia enfoques dinámicos e integrales, y no sólo de tipo comportamental y psicopatológico, así se consideró contribuir a la comprensión de la manera en que se produce el desarrollo personal de los adolescentes, y el lugar que ocupa la resiliencia en ellos desde su propia perspectiva, en jóvenes que se ven enfrentados a un mundo complejo, de riesgos y oportunidades, donde la violencia es una clara característica. Se hizo una investigación cualitativa, con un enfoque hermenéutico; se realizaron 48 entrevistas en profundidad, las cuales se analizaron con herramientas de la teoría fundamentada en tres etapas, descriptiva, analítica e interpretativa. Se encontró que la clasificación taxativa de los jóvenes como resilientes o no, o con problemas de conducta o no, no corresponde a cómo ellos viven su desarrollo, este se da de manera dinámica y cambiante, de acuerdo con sus características, potencialidades, necesidades e intereses, y las oportunidades que encuentran en los contextos.

Desarrollo del Adolescente; Asunción de Riesgos; Resiliencia Psicológica; Violencia

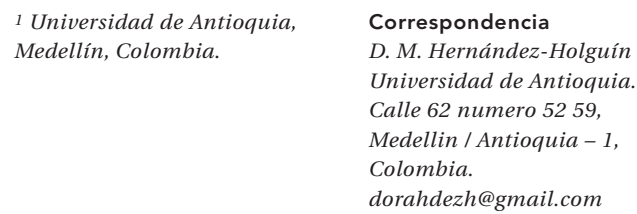




\section{Introducción}

Pese a la importancia de los estudios de prevalencia, que han permitido identificar la magnitud de conductas de riesgo 1,2 y de resiliencia en la población joven de Medellín, Colombia 3; como alternativa teórica, que muestra la complejidad en relación con los comportamientos, se tiene la perspectiva contextual evolutiva, que concibe el desarrollo humano desde un modelo integrado de las relaciones indivíduo-contexto, y no solamente desde las características del organismo. Esta concepción de desarrollo considera que la interacción de la persona con el ambiente es activa y bidireccional, lo que conlleva una visión más dinámica e integral del joven, en términos de su plasticidad e interacción con los diferentes niveles (micro, meso, macro) del contexto, con lo que llama la atención hacia estudios más sensibles a mostrar la connotación dinámica del desarrollo 4,5,6.

Teniendo en cuenta lo anterior, y como se puede ver más adelante, los jóvenes de Medeliín se mueven entre la inequidad, la violencia y las oportunidades, y aunque muchos presentan claros comportamientos de agresión severa, conductas sexuales de riesgo y/o uso de sustancias psicoactivas, como lo muestran los estudios poblacionales disponibles; también en muchos de ellos sobresalen conductas resilientes, lo que motiva a avanzar en el estudio sobre la manera en la que los jóvenes se van desarrollando, al pasar de una mirada centrada en la psicopatología y en los factores de riesgo, a una concepción más compleja y dinámica del joven desde su propia perspectiva.

El desarrollo positivo es una de las perspectivas de estudio de la resiliencia, cuyo objetivo es promover cualidades en un individuo, que le permitan superar la adversidad 7 . Algunas de estas cualidades están relacionadas con auto-regulación emocional y comportamientos que contribuyen a la salud mental y al buen rendimiento académico ${ }^{8}$, lo que indica un punto de partida desde el cual se concibe al joven y su desarrollo personal de manera dinámica, en relación con su contexto social y cultural, el cual juega, entonces, un papel importante en las vivencias de un proceso resiliente, en relación con ello, la resiliencia, en palabras de Ungar 9 (p. 225), es "la capacidad individual y colectiva de navegar en dirección a los propios recursos psicológicos, sociales, culturales y físicos para su bienestar; y a su vez, la capacidad de poner a disposición tales recursos de una forma que tenga sentido en la cultura". Desde esta perspectiva, se sugiere más investigación sobre resiliencia en niños y adolescentes en los países de medianos y bajos ingresos 10 , de manera que -sin negar la resiliencia como capacidad- también está presente su connotación dinámica y la posibilidad de promoción de resiliencia en la interacción permanente con los jóvenes.

En cuanto al contexto social de los jóvenes que participaron en la investigación, sobresale la inequidad social 11. Medellín, ciudad aún afectada por la violencia 12 , con más de dos millones de habitantes, y cerca de 285.000 jóvenes de 13 a 20 años, la mitad de ellos de estrato socioeconómico bajo ${ }^{13}$, es una ciudad de contrastes, la educación media, aunque con altibajos, se ratificó en 2013 con $79,2 \%$ en cobertura educativa y tras una cobertura creciente en educación superior, para 2012 cerró en una tasa bruta de 53,2\% 14; no obstante, en calidad de la educación básica, el 45,5\% de los estudiantes no alcanzaron el nivel mínimo en lectura, y en matemáticas el $67,2 \% 12$, lo que muestra coherencia con el Índice de Desarrollo Juvenil (72,5\%) 15 en el que hay mayores logros en el acceso a bienes y servicios que en el desarrollo de los jóvenes, las dimensiones de mayor aporte son educación (19), bienes y servicios $(16,8)$ y salud (11,2); y las que menos aportan: desarrollo del ser del joven $(3,9)$, democracia y participación $(2,9)$ y trabajo decente $(0,8)$.

En el estudio de esta realidad ha predominado un enfoque de riesgo, lo que ha permitido identificar la magnitud de problemas de comportamiento y factores asociados a ellos. Con respecto a la violencia, el recrudecimiento de esta en 2008 y 2009 significó incrementos de homicidios del 34\% entre 2007 y 2008 y mayor al 100\% entre 2008 y 2009, hasta llegar este último año a 94 homicidios por 100 mil habitantes 12 y ha venido disminuyendo hasta llegar a 26,9 en 2014 16. Se ha encontrado también que alrededor del $11 \%$ de los jóvenes presenta agresión severa 17,18, mientras la prevalencia en el último año para consumo de bebidas alcohólicas hasta la embriaguez pasó de $19 \%$ a $23,2 \%{ }^{2}$ y la edad media de inicio de relaciones sexuales es de 14 años 17 .

En cuanto a problemas afectivos, la prevalencia de depresión pasó del 12,1\% en el 2006 al $13,1 \%$ en el 2009 2; como indicadores de ideación suicida, un $8,9 \%$ de los adolescentes casi siempre se quiere morir, y el $3,5 \%$ alguna vez ha intentado suicidarse y requirió atención en salud por este evento 2 ; el $24,7 \%$ presenta estrés postraumático crónico leve, el 25,3\% moderado y el $34,5 \%$ severo 1,18 .

Pocos estudios en la ciudad han aportado información sobre desarrollo positivo del joven, en uno de ellos, encontraron como factores asociados a la resiliencia: hogares nucleares, con padre y madre, madre vigilante y con quien podían tener una buena comunicación ${ }^{19}$. Otro estudio reportó como jóvenes resilientes, el 22,9\% de los 
encuestados 3 , y en un tercer estudio -en un contexto similar- refiere la importancia de fortalecer las interacciones familiares, y el soporte comunitario, en términos de afecto e interacción social positiva que brinde información y experiencias formativas, en beneficio de la salud mental de los jóvenes 20.

Esta investigación permite entonces contribuir hacia la comprensión de la manera como se da el desarrollo personal de los adolescentes, principalmente en cuanto a su carácter dinámico -en interacción con su contexto- no sólo sobre la presencia o no de sus comportamientos de riesgo -y el lugar que ocupa la resiliencia en él.

\section{Materiales y métodos}

Se utilizó un enfoque histórico hermenéutico desde el cual se concibe al ser humano como un intérprete de la realidad que vive y construye a cada momento 21 ; un diseño cualitativo 22 y teoría fundamentada, como método de investigación, la cual tiene como soporte teórico, los postulados del interaccionismo simbólico 22.

Participaron 48 jóvenes de 13 a 20 años, con residencia de tres años o más en barrios de estratos socioeconómicos bajos. Estos jóvenes se identificaron de un estudio previo de casos y controles, en el que además de una clasificación por conductas de riesgo, también se midieron conductas resilientes 22; de él se entrevistaron a 48 jóvenes, 16 con características resilientes, 16 con conductas de riesgo como consumo y/o tráfico de sustancias psicoactivas, trabajo sexual, agresiones, robo, asesinato, vinculación a grupos armados, y 16 que no presentaban conductas resilientes, pero tampoco sobresalían en ellos conductas de riesgo. En un muestreo guiado por la teoría, y paralelo al análisis de la información los jóvenes, se fueron convocando de 4 en 4 por cada grupo, hasta llegar a la saturación de la categoría "la resiliencia como un proceso posible y constante en los jóvenes”. Es importante aclarar que la clasificación aportó inicialmente en heterogeneidad en el grupo de jóvenes, pero fue perdiendo sentido a lo largo de la investigación, como se aclarará en los resultados. La participación de los jóvenes fue voluntaria, sin incentivo material por ello, se les reconoció transporte cuando era necesario y refrigerio.

Cada entrevista fue orientada por los investigadores, todos, profesionales de las ciencias sociales, algunos, con posgrado en salud pública y salud mental, y con experiencia en investigación cualitativa y en el trabajo con jóvenes, además, se realizaba en el lugar elegido por el joven. La entrevista siguió la línea de vida del joven, como orientación cronológica de sus experiencias significativas, tanto personales, como de acceso a servicios. El contenido de la entrevista era grabado, transcrito, y codificado con herramientas de Microsoft Office (Microsoft Corp., EE.UU.).

El análisis, simultáneo a la recolección, fue en tres fases: descriptivo, analítico e interpretativo, con 24, 16 y 8 entrevistas, respectivamente. A partir del primer análisis, mediante codificación abierta, se construyó el libro de códigos, y emergieron 39 categorías descriptivas y 251 subcategorías. La codificación abierta y la emergencia de categorías descriptivas permite llegar a conceptos, ideas y significados; así los datos se descomponen, se comparan entre sí y se agrupan en lo que se llamarán "categorías descriptivas" 23.

Luego, se realizó una codificación axial, por medio de la cual se agruparon y relacionaron las categorías descriptivas. Este ejercicio es el inicio de la formulación teórica y la agrupación de las categorías, que se orienta según las relaciones entre categorías, tanto de aquellas que hacían alusión a la resiliencia, como al contexto y la acción e interacción de los sujetos con dicho fenómeno ${ }^{23}$; emergieron las categorías analíticas: condiciones de riesgo, auto-percepción, motivaciones e intereses, estrategias de afrontamiento, actitudes hacia la vida y actitudes hacia el servicio.

En la tercera fase, por medio de codificación selectiva y mapas conceptuales, estos últimos como "herramientas gráficas de asociación, interrelación, discriminación, descripción y ejemplificación de contenidos, con un alto poder de visualización, que permite organizar y expresar las ideas, comprender y clarificar conceptos" 24 , se integraron y refinaron las categorías identificadas, hasta llegar a la categoría interpretativa "la resiliencia como un proceso posible y constante en los jóvenes", en la cual caben las características de resiliencia propias de los jóvenes entrevistados y su carácter dinámico y de interacción con el medio.

En todo el proceso se tuvieron en cuenta los aspectos éticos, entre estos, cuando el joven era mayor de edad firmaba el consentimiento informado y, si era menor, previo al asentimiento del joven, los padres firmaban el consentimiento; se aseguró la confidencialidad de la información, para el reporte de los hallazgos, los códigos de las entrevistas fueron usados en cada caso para proteger la identidad de los entrevistados. Todo ello, siguiendo las pautas de la Resolución no 8.430 de 1993 del Ministerio de Salud de Colombia 25 y los criterios de la Declaración de Helsinki para la protección de la vida, la salud, la dignidad e integridad de los entrevistados. El estudio contó con el aval del Comité de Ética del Centro de Investigación de la Facultad Nacional de Salud Pública. 


\section{Resultados}

La resiliencia como un proceso posible y constante

Como resultado de la investigación, la resiliencia se concibe como un proceso en el que el joven a lo largo de su vida se va haciendo más o menos resiliente. La resiliencia se explícita dependiendo de cada experiencia que viva el joven y de qué tan significativa sea esta para él o ella, es decir, cada experiencia vivida en interacción con las características del joven en ese momento (potencialidades, necesidades, intereses) puede generar, en alguna medida, la gestión de diferentes recursos del joven y de su entorno para un beneficio propio, o al contrario, lo puede llevar a adoptar actitudes y comportamientos que van en desmedro de su bienestar y desarrollo personal, y con ello, de quienes lo rodean.

El contexto toma relevancia para el proceso de resiliencia en el joven al proveer recursos, principalmente familiares, para enfrentar las dificultades a lo largo de su vida, en los mejores casos, en su aprendizaje para el manejo positivo de estas situaciones. Todo ello va marcando formas de ser y de vivir de los jóvenes, sus particularidades, intereses, carácter y satisfacción con la vida.

"Por ahí hace poquito tuve un problema con un vicio y sí, mi mamá me aconsejó y entonces yo me sali" (E41).

"Mi hermana ha sido un apoyo en el estudio... Se preocupa por nosotros, es la que me sacó adelante, desde pequeño, dándome los estudios, todo ella. Ha sido el apoyo básico, también por ella dejé casi todo atrás, el círculo en que estaba [las drogas]. Por ella, me di cuenta que fue un círculo... yo me puse a pensar lo que hacía: 'trábese y póngase a mirar a los demás... y era verdad lo que ella decía" (E39).

"Si yo estudio es por mi papá y mi mamá, porque ellos son los que lo motivan a uno, uno hace las cosas no sólo por uno, sino por la motivación que los demás le den" (E44).

Cuando la interacción del joven con su medio facilita el desarrollo de características de resiliencia, estas se pueden explicitar en el establecimiento de metas y objetivos hacia una superación personal, a partir de la búsqueda del bienestar propio, de su familia y su comunidad, es decir, una actitud y actuación hacia un bienestar propio y común, bajo un claro componente moral. En este sentido, en el discurso de los jóvenes emergieron diferentes características de resiliencia, a pesar de que algunos de ellos incurren en conductas de riesgo.

"Sí, yo tengo mi proyecto de vida, yo me imagino pues organizándole acá la casa a mi mamá, me imagino teniendo a mi mamá a lo bien; yo ser una profesional... Y ayudarle a la gente que lo necesita, así me imagino" (E25).

"...Siempre me dicen, usted tiene que estudiar para que pueda salir adelante, entonces es un apoyo, que uno dice, , claro!, uno tiene que estudiar para también gratificar lo que ellos le han dado a uno, uno salir adelante y ayudarlos también" (E18).

En el proceso de resiliencia, los jóvenes que incurren en mayor medida en conductas de riesgo, como aquellas relacionadas con el fracaso escolar, la violencia, las drogas y la sexualidad insegura, también relatan cómo cuando hay un deseo de cambio y de superación, y una clara ganancia al transformar esas acciones que van en contra de su bienestar, ponen todas sus capacidades en pro del cambio, y del alcance de metas personales, dejando de lado las conductas riesgosas.

Este deseo de cambio no está presente solo en los jóvenes con conductas claras de riesgo, sino también en los que incurren en menor medida en ellas. Para ambos los eventos importantes, como nacimiento de un hijo, una experiencia muy cercana con la muerte o la llegada de un adulto significativo -ya sea de la familia, la comunidad o un servicio-, marca una diferencia entre los comportamientos anteriores y la manera como se empieza a asumir la vida en adelante.

"[Abuso sexual, indiferencia de la madre]... Esas son las que más me han marcado y otras más, muchas que me han hecho sufrir, que prefiero olvidarlas porque ya voy a ser mamá, tengo una persona que me ama, y me ha hecho olvidar muchas tragedias" (E21).

“Duro porque es la muerte de cuatro amigos... y si pues, esas cosas hacen que uno reflexione porque uno ya empieza a ver como qué puede pasar.. y darme cuenta de que tengo que cambiar, tengo que mejorar, porque si no, voy a terminar igual [asesinado]" (E44).

\section{Algunas diferencias entre los jóvenes}

Un contexto social y familiar difícil, relacionado con la pobreza, la violencia y el abuso sexual, es lo común entre los jóvenes entrevistados, ya sea con mayor o menor cercanía a la propia vida, pero es un contexto que presenta posibilidades de riesgo a cada paso, y aunque hay algunas características similares entre los jóvenes, sobresalen de manera clara aquellas diferencias individuales con respecto a la resiliencia (Figura 1).

Los jóvenes que muestran más resiliencia tienen una imagen positiva de sí mismos y se consideran eficaces, lo que les permite tener confianza, seguridad y éxito en los diferentes proyectos 


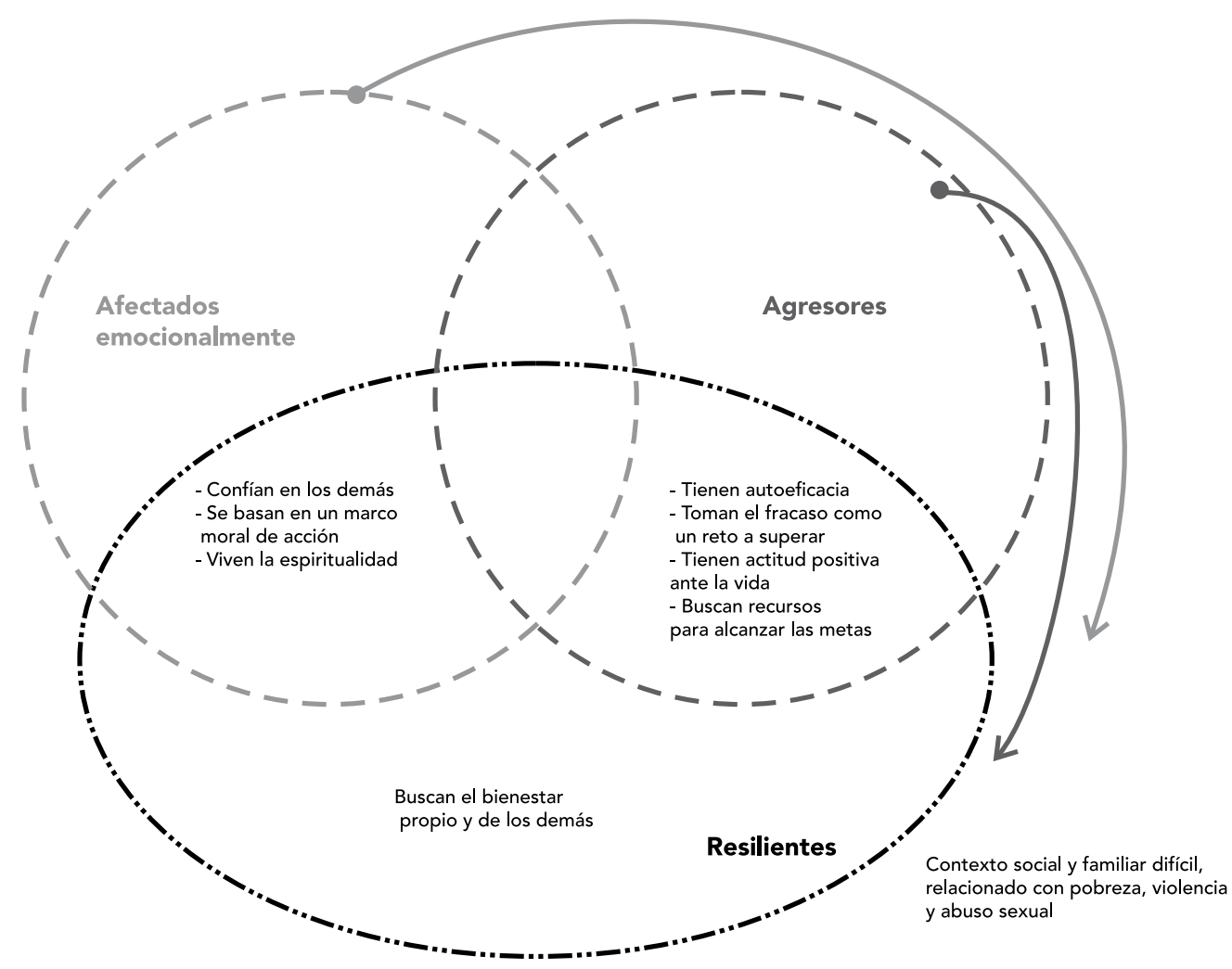

que emprenden. Cuando estos jóvenes enfrentan el fracaso lo visualizan como retos a superar o pruebas que les ha puesto la vida para salir de allí fortalecidos. Esto último se encuentra relacionado con esa capacidad que tienen los jóvenes con conductas resilientes de ver oportunidades y posibilidades, donde otros no las ven, y de encontrar soluciones en lugar de quedarse en la queja y en el problema.

Otro elemento importante que les caracteriza es su capacidad para reflexionar, acerca de las metas y objetivos que desean alcanzar, para algunos sus metas están en el ámbito académico, para otros en el ámbito deportivo, artístico e inclusive en lo laboral, con el fin de mejorar las condiciones económicas propias y de su familia. Lo más importante de estas es que no son metas desarticuladas de la realidad o imposibles de llegar a cumplir, al contrario, son metas realizables, es decir, que tienen asiento en la realidad; metas que el joven visualiza como capaz de lograr, y se dispone a una constante búsqueda y gestión de recursos para alcanzarlas.
La búsqueda de recursos para alcanzar las propias metas se manifiesta en una mayor participación de los jóvenes en los servicios que se ofrecen en la comunidad y/o colegio, allí pueden encontrar formas para solucionar dificultades, capacitarse académica y laboralmente, hallar personas de confianza que en momentos de dificultad le puedan dar apoyo emocional o económico. Pero no sólo en los servicios el joven busca recursos, también en su familia, o en las personas cercanas de su comunidad, en quienes puede encontrar ayuda para el logro de sus metas.

De esta forma, tanto en los servicios, como en la comunidad y la familia el joven encuentra recursos para su desarrollo personal, sin embargo, esta relación no es unidireccional, por el contrario, en diferentes ocasiones el joven despliega allí todas sus capacidades, convirtiéndose en un líder que trata de que los escenarios donde se desenvuelve mejoren. Este trabajo en pro de la comunidad refleja igualmente el marco moral, dentro del cual actúa el joven con mayores características resilientes, un marco moral que busca 
el logro de objetivos y metas sin pasar por encima del bien común, sin vulnerar al otro. Otro elemento clave es la auto-eficacia, la capacidad para enfrentar los problemas: actuar, evitarlos, huir o reflexionar, según como sea necesario y hallar la solución más adecuada.

“...Eso yo me lo gané con mucho esfuerzo porque, si usted supiera qué es ir a un consejo comunal a pelear con todos esos viejitos y esas señoras, peleando, por la cera de la casa, con las feministas ijaa!, que obras públicas es más importante que educación, que para qué les iban a dar educación a esos muchachos que no querían estudiar, eso era una pelea horrible, entonces yo creo que eso me lo gané merecidamente [la aprobación de presupuesto para educación superior de jóvenes de la comuna]" (E9).

"En vez de retroceder, me estoy sintiendo adelante, por ejemplo, me va bien en la universidad, y aunque tengo problemas... tuve que faltar a clase por mi baby, voy siguiendo y si me toca entregar los trabajos ahí mismo, voy y los traigo, entonces le voy dando también una solución a eso... voy pa' delante, voy primero, segundo y tercer semestre sin quedarme en ninguna materia. En mi casa las cosas están bien, aunque no hay que negar que lo económico es un problema, pero siempre se le busca solución a las cosas, que ya tengo mi casa, o sea, que ya estoy progresando, que ya me estoy independizando" (E26).

Este afrontamiento efectivo realimenta la confianza y la imagen positiva de sí mismo. Sumado a ello está la actitud positiva frente a la vida y las dificultades, y la espiritualidad que les hace fuertes, tener esperanza y fe en que las dificultades se pueden superar. Es importante aclarar que esta espiritualidad no se refiere a una posición pasiva del joven, desde la que todo se lo atribuye a Dios y espera que este solucione sus obstáculos, sino por el contrario, una fuerza que orienta y tranquiliza frente a lo que pueda llegar (Figura 1).

“...Para mí lo más importante es Dios, en mi vida el primer lugar lo ocupa Dios y eso nadie me lo va a quitar.. yo quiero que la gente conozca a Dios, yo quiero que sepan, por ejemplo los jóvenes... que en la iglesia también se vive, que la iglesia se siente" (E27).

Algunos jóvenes aunque presentan conductas resilientes, también incurren en conductas de riesgo, como consumo de drogas, deserción escolar y relaciones con pares que igualmente son consumidores o pertenecen a grupos ilegales, conductas que se constituyen en posibles opciones de adaptación. Estas conductas de riesgo no siempre son reconocidas por el joven como tal, por el contrario las normaliza, lo que se relaciona con su actitud pasiva frente a la vida y con la forma como ellos tramitan sus emociones; emocio- nes que generalmente son de carácter negativo y emergen como consecuencia de vivencias de eventos significativos que vulneran al joven, como la separación de los padres, abusos sexuales, abortos, violencia en la familia, dificultades económicas. Estos jóvenes se describen como solitarios, desconfiados y callados, y en su discurso se manifiesta constantemente frustración consigo mismos y con sus vidas.

Las estrategias de afrontamiento más relevantes de estos jóvenes son búsqueda de ayuda en otras personas, generalmente en quienes confían como la madre o profesores cercanos, esta ayuda es principalmente de tipo afectiva o apoyo emocional, sin embargo, algunos de ellos no logran encontrar ese apoyo. Otra estrategia es reflexionar acerca de posibles alternativas de solución, aunque muchos prefieren huir o evadir, esperando que los problemas se solucionen solos, estrategia que en muchas ocasiones tiene como consecuencia que el problema se intensifique, momento en el que han adquirido relevancia las características y oportunidades que ofrecen los adultos que rodean a estos jóvenes, para promover en ellos la resiliencia (Figura 1).

“Me gustaría sacar adelante unos proyectos que tengo, pero lo que pasa es que la situación económica no me da, y... no veo el apoyo de mis padres, entonces uno en el mundo es como si fuera solo, no encuentro apoyo de nadie sino en mí solo... Por eso uno se mantiene así inconforme, tranquilo [actitud pasiva]" (E14).

“...Ella [adulta cercana] me apoyó mucho cuando veía que yo tenía problemas en mi casa... ella hizo que ese dolor no lo sintiera tan agudo.. yo era muy depresiva" (E21).

Otros jóvenes, con claros comportamientos de riesgo, relatan cómo afrontan sus dificultades, algunos deciden consumir sustancias psicoactivas, vincularse a grupos armados ilegales y/o evadir la situación. Estos comportamientos en sí mismos y por sus consecuencias no van en línea con un bienestar y desarrollo saludable, dado que llevan encadenadas otras conductas o consecuencias como bajo rendimiento académico, deserción escolar, sexualidad insegura, embarazos no deseados, aislamiento social, desplazamiento intraurbano por amenazas a la integridad física, e incluso la muerte.

La razones que dan estos jóvenes para incurrir en conductas de riesgo están relacionadas con lograr un reconocimiento social, cuando se trata del ingreso a bandas delincuenciales, curiosidad o presión de pares, cuando se trata de consumo de sustancias, y escape emocional, cuando se trata de consumo de alcohol; se denota en sus discursos cierta ambición y un deseo de poder y dinero, lo que hace que el joven sea fácilmen- 
te influenciable, es decir, que sucumba ante la presión de otros, con el fin de obtener prestigio y/o bienes materiales. En su vida cotidiana, estos jóvenes manifiestan abiertamente sus intereses personales, y muestran su eficacia por medio del alcance de metas, pero en estas no se hace visible la "reflexión moral", vacío que puede llevarlos a aceptar la participación en grupos armados o consumir drogas, pero también podría constituirse en propósito de formación por parte de los adultos que acompañan al joven (Figura 1).

“i...Por qué un joven en esta situación conflictiva entra a hacer parte de estos combos? Pues... no sé cómo decirle, como una forma más de salir adelante" (E42).

“...Uno vivir en un barrio, un barrio bajo... Usted sale a la esquina y vemos a alguien fumando marihuana, eso es como lo más normal... a mí me parecía que era normal, y ahí quedé... imagínate por lo normal pasé por frío, aguanté hambre... Mi hermana me ayudó a entender" (E39).

\section{Discusión}

La resiliencia, como proceso permanente en la vida de los jóvenes, se logra cuando estos tienen las oportunidades de acuerdo con sus características particulares, el joven con evidentes características resilientes recibió esta influencia y claridad desde los primeros años, y va tras de sus sueños; los demás jóvenes lo van logrando en la medida en que quien les ayuda en el proceso sabe llegar a las "emociones" en algunos, y en otros propicia una "reflexión moral y práctica" de las ganancias de un comportamiento saludable.

Uno de los elementos que permite ver el desarrollo de los adolescentes entrevistados como un proceso complejo y dinámico, más allá de la configuración de estructuras psicopatológicas o de la descripción de conductas, tiene que ver con la relación bidireccional y profunda entre el adolescente y sus diferentes niveles de interacción -lo que incluye las características propias de su edad, los desafíos (de cambio corporal, identidad, académicos, sociales) que le demanda cada contexto, y las características de cada interacción que establece, la influencia de pares, los padres y la comunidad-lo que puede verse más claramente una vez que el joven esté presentando un desarrollo saludable, o en cambio, cuando ya está presentando diversas conductas de riesgo 26,27.

Se pudo ver en los jóvenes entrevistados, que a pesar de que el adolescente esté presentando conductas de riesgo, en este periodo de transición y de cambios, se pueden hacer realidad las expectativas de un desarrollo saludable, siempre que las oportunidades para lograrlo se pongan a su servicio 26, máxime que se ha documentado la importancia de que el joven conciba su futuro como esperanzador 28 , referido a la posibilidad de lograr una vida satisfactoria. Desde su contraparte, en Medellín, también se ha encontrado, cómo la frustración de expectativas de desarrollo personal (estudiar, formar una familia) constituye un factor de riesgo para la violencia 17 .

Lo anterior lleva a considerar la importancia de identificar aquellas características, potencialidades, necesidades, intereses, propias de cada adolescente, de manera que se pueda proponer una interacción que lo favorezca a él y a su entorno, hacia un desarrollo positivo; de tal manera que conviene reconocer en cada joven cómo va su proceso de resiliencia, y lo que requiere para alcanzar sus metas, ya sea lograr una autorregulación emocional, la identificación misma de sus intereses, una reflexión moral sobre sus acciones o la gestión de recursos para lograr sus propósitos.

El desarrollo positivo, como perspectiva teórica, ha aportado el constructo de las cinco "C", para evaluar el desarrollo de los adolescentes: la competencia, es una visión positiva de sí mismo, en lo social, académico, cognitivo y profesional; la confianza, sentido interno de la auto-eficacia y autoestima de manera general; el carácter implica el respeto de las normas sociales y culturales, tener un sentido de lo correcto y lo incorrecto; la conexión implica un vínculo positivo con las personas e instituciones, que se reflejan en intercambios bidireccionales saludables entre el individuo y los compañeros, la familia, la escuela y la comunidad; el cuidado, es el grado de simpatía y empatía que el adolescente siente hacia los demás 28,29. Características similares a las halladas en los jóvenes entrevistados, en las que cabe resaltar: auto-eficacia, confianza en los demás, actitud positiva ante la vida, marco moral de acción, y búsqueda del bienestar propio y el de los demás.

El desarrollo de los jóvenes no se manifiesta como una línea recta e inmodificable, por el contrario, está lleno de altibajos en los que las características personales que se van formando influyen directamente en los diferentes comportamientos, actitudes y creencias que el joven puede asumir. Los comportamientos de los jóvenes pueden estar orientados hacia la búsqueda del bienestar propio y de los que le rodean, o también ir en pro de conductas de riesgo, que lo perjudican a él o a su entorno, más aún, puede verse que los mismos cambios en el joven no son lineales ni absolutos, al contrario son dinámicos, lo que podría indicar que si se mantiene una forma de acompañamiento cercana, que respete su individualidad, su desarrollo será continuo, así las cosas, los jóvenes refirieron superación de 
consumo de drogas, mejoramiento académico, y superación de secuelas de abuso sexual.

Para lograr lo anterior, aunque no todas las estrategias de afrontamiento son igualmente eficaces, pareciera que la búsqueda de apoyo social y la solución activa, conductual o cognitiva del problema son estrategias que juegan un papel importante en el proceso de desarrollo de la resiliencia 30 , al contar con estrategias activas de afrontamiento, se tienen más probabilidades de experimentar menos estrés, en cambio la negación del problema, la inactividad, el consumo de alcohol y drogas, el hacerse ilusiones y el autoinculparse forman parte de las estrategias negativas o ineficaces 31 .

Contribuir hacia una mayor comprensión del desarrollo personal y del desempeño de los adolescentes, como un proceso complejo y así mismo dinámico, se justifica, no sólo por el conocimiento en sí mismo, sino por las acciones que desde el sector público, clínico y en los espacios cotidianos, como la familia o la escuela, se puedan emprender con y para los jóvenes. Los profesionales que trabajan en programas y servicios dirigidos a los adolescentes y jóvenes pueden tener una mayor comprensión de las posibilidades que tienen los jóvenes para su desarrollo saludable, según sus particularidades, de manera que se apoye la gestión de recursos cuando el joven tiene claro su proyecto de vida, pueda contar con la orientación para superar sus barreras emocio- nales, o se le acompañe en el reconocimiento de las implicaciones morales de sus actos, y en todos los casos, se les ayude a clarificar sus metas personales, construir las alternativas y gestionar los recursos disponibles para alcanzarlas.

A manera de conclusión, toman relevancia las metas que establecen los jóvenes para su futuro, como una manera de concebir posible dinamismo en su desarrollo personal. Una mirada en contraste de las características, tomando en consideración el tipo de conductas de los jóvenes, nos muestra que aquellos con conductas violentas y resilientes, comparten cualidades activas ante la vida, mientras los jóvenes que no son resilientes, ni agresores, tienen en general una actitud pasiva, con expresa afectación emocional, aunque no se incurran en conductas de riesgo. De igual manera, estas características personales de los jóvenes no son estáticas ni exclusivas de un tipo de joven, se van modificando de manera dinámica, lo que da opciones para la promoción de la resiliencia.

El estudio realizado, aunque muestra claramente el dinamismo en el desarrollo de los jóvenes y la resiliencia como un proceso constante y posible, no permite comprender el carácter integral del desarrollo, en las interacciones de los jóvenes con los diferentes niveles (micro, meso y macro) del contexto de manera específica, especialmente de tipo familiar, pero también institucional y comunitario, con que el joven interactúa.

\section{Colaboradores}

D. M. Hernández-Holguín orientó el análisis e interpretación de los datos, escribió la introducción y la discusión de resultados. Se hizo responsable de todo el proceso de escritura y publicación del artículo, hasta la aprobación final de la versión a ser publicada. I. C. Sánchez apoyó el análisis e interpretación de datos y la redacción de los resultados, revisó versiones finales del artículo. E. Páez participó en el análisis e interpretación de los resultados, revisó versiones finales del artículo. E. M. Montoya-Vásquez participó en el análisis e interpretación de los resultados y las versiones finales del artículo revisado.

\section{Agradecimientos}

Al equipo de investigación del Proyecto Pathways Towards Resilience de Medellín, por su aportes en la base de datos para la identificación de los jóvenes entrevistados, al equipo de investigación cualitativa, por su relevante apoyo en la discusión del proyecto y en la recolección de la información, así como al Profesor Michael Ungar, investigador principal, por su revisión del artículo. A International Development Research Centre (Dalhousie U) Estrategia Sostenibilidad GISAME UdeA 2014 por la financiación. 


\section{Referencias}

1. Torres Y, Osorio J, López P, Mejía R. Salud mental del adolescente, Medellín 2006. Medellín: Alcaldía de Medellín/Universidad CES; 2007.

2. Montoya L, Torres Y, Zapata M, Garro G, Hurtado G. Segundo estudio de salud mental del adolescente - Medellín. Revista Salud Pública de Medellín 2010; 4:23-40.

3. Duque LF, Montoya NE, Restrepo A. Aggressors and resilients youths in Medellín, Colombia: the need for a paradigm shift in order to overcome violence. Cad Saúde Pública 2013; 29:2208-16.

4. Lerner RM, Lerner JV, Phelps E. The positive development of youth. Technical report. The 4-H study of positive youth development: report of the findings from the first four waves of data collection: 2002-2003, 2003-2004, 2004-2005, and 2005-2006. Boston: Institute for Applied Research in Youth Development, Tufts University; 2008.

5. Lerner RM, Lerner JV, Bowers EP, Geldhof GJ. Positive youth development and relational-developmental-systems. Hoboken: John Wiley \& Sons; 2015.

6. Gestsdottir S, Lerner R. Intentional self-regulation and positive youth development in early adolescence: findings from the 4-H Study of Positive Youth Development. Dev Psychol 2007; 43:508-21.

7. Rutter M. Implications of resilience concepts for scientific understanding. Ann N Y Acad Sci 2006; 1094:1-12

8. Goldstein S, Brook R, editors. Handbook of resilience in children. New York: Springer; 2006.

9. Ungar M. Resilience across cultures. Br J Soc Work 2008; 38:218-35.

10. Kieling C, Baker-Henningham H, Belfer M, Conti G, Ertem I, Omigbodun O, et al. Child and adolescent mental health worldwide: evidence for action. Lancet 2011; 378:1515-25.

11. Misión para el Empalme de las Series de Empleo. Nueva metodología para la medición de la pobreza monetaria y cifras de pobreza extrema, pobreza y desigualdad 2002-2010. Bogotá: Departamento Nacional de Planeación/Departamento Administrativo Nacional de Estadística; 2011.

12. Medellín cómo Vamos. Seguridad ciudadana 2014 http://www.medellincomovamos.org/seguridady-convivencia (accedido el 25/Oct/2015)

13. Departamento Nacional de Estadística. Proyecciones de población. Estimación y proyección de población nacional, departamental y municipal por sexo, grupos quinquenales de edad y edades simples de 0 a 26 años 1985-2020. http://www.dane. gov.co/index.php?option=com_content\&view=art icle\&id=75\&Itemid=72 (accedido el 28/Feb/2014).

14. Medellín cómo Vamos. Informe de educación, 2014 http://www.medellincomovamos.org/educacioncultura-y-recreacion (accedido el 25/Oct/2015).

15. Alcaldía de Medellín. Índice de desarrollo juvenil y línea de base 2011-2012. Medellín: Alcaldía de Medellín; 2012.

16. Consejo Ciudadano para la Seguridad Pública y Justicia Penal. Pelo quarto ano consecutivo, San Pedro Sula ocupou o primeiro lugar mundial. http://www.seguridadjusticiaypaz.org.mx/biblio teca/prensa/send/6-prensa/200-as-50-cidadesmais-violentas-do-mundo-em-2014 (accedido el 27/Oct/2015)
17. Duque L. La violencia en el Valle de Aburrá. Caminos para la superación. Medellín: Cátedra Litográfica; 2007.

18. Torres Y, Zapata M, Montoya L, Garro G, Hurtado G. Segundo estudio de salud mental del adolescente. Medellín: Alcaldía de Medellín/Universidad CES; 2009.

19. Duque L, Klevens J, Montoya N. Conductas socialmente indeseables asociadas a agresores y resilientes. Un estudio de casos y controles en $\mathrm{Me}$ dellín, Colombia. 2003-2005. Rev Fac Nac Salud Pública 2007; 25:21-36.

20. Benetti SPC, Schwartz C, Soares GR, Macarena F, Pattussi MP. Psychosocial adolescent psychosocial adjustment in Brazil: perception of parenting style, stressful events and violence. Int J Psychol Res (Medellin) 2014; 7:40-8.

21. De La Maza LM. Fundamentos de la filosofía hermenéutica: Heidegger y Gadamer. Teología y Vida 2005; 46:122-38.

22. Montoya NE, Restrepo A, Duque LF, Ungar M. Predicting resilient adolescents and youths: validation of screening test in Colombia. Issues Compr Pediatr Nurs 2011; 34:167-88.

23. Strauss A, Corbin J. Bases de la investigación cualitativa. Técnicas y procedimientos para desarrollar una teoría fundamentada. Medellín: Editorial Universidad de Antioquia; 2002.

24. Vidal Ledo M, Febles Rodríguez P, Estrada Sentí V. Conceptual maps. Educ Med Super 2007; 21(3). http://scielo.sld.cu/scielo.php?script=sci_arttext\& pid=S0864-21412007000300011\&lng=es.

25. Ministerio de Salud. Resolución 8430 de octubre 4 de 1993. Normas científicas, técnicas y administrativas para la investigación en salud. Bogotá: Ministerio de Salud; 1993.

26. Theokas C, Lerner R. Observed ecological assets in families, schools, and neighborhoods: conceptualization, measurement, and relations with positive and negative developmental outcomes. Appl Dev Sci 2006; 10:61-74.

27. Rutter M. Resilience as a dynamic concept. Dev Psychopathol 2012; 24:335-44.

28. Schmid KL, Phelps E, Lerner RM. Constructing positive futures: modeling the relationship between adolescents' hopeful future expectations and intentional self regulation in predicting positive youth development. J Adolesc 2011; 34:1127-35.

29. Lerner R. Promoting positive youth development: theoretical and empirical bases. Medford: Tufts University; 2005.

30. Ruus V, Veisson M, Leino M, Ots L, Pallas L, Sarv S, et al. Students' well-being, coping, academic success, and school climate. Soc Behav Pers 2007; 35:919-36.

31. Ahern N, Ark P, Byers J. Resilience and coping strategies in adolescents. Paediatr Nurs 2008; 20:32-6. 


\section{Abstract}

Research on young people has increasingly focused on dynamic and comprehensive approaches rather than merely behavior and psychopathology. Such studies help shed light on personal development in adolescents and the role of resilience for them from their own perspective, that is, young people facing a complex world of risks, opportunities, and omnipresent violence. A qualitative study was performed with a hermeneutic focus; 48 in-depth interviews were performed, analyzed with tools from the three-stage framework (descriptive, analytic, and interpretative). The results show that all-or-nothing classification of youth as resilient versus non-resilient (or "with" versus "without" behavior problems) fails to reflect how they experience their development, which is dynamic and changes according to their characteristics, potentialities, needs, and interests, as well as the opportunities they find in their contexts.

Adolescent Development; Risk-Taking; Psychological Resilience; Violence

\section{Resumo}

As pesquisas sobre jovens têm se concentrado cada vez mais em abordagens dinâmicas e integradas, em vez de apenas no comportamento e na psicopatologia. Tais estudos ajudam a elucidar o desenvolvimento pessoal dos adolescentes e o papel da resiliência para eles próprios, partindo da sua própria perspectiva, isto é, de jovens que enfrentam um mundo complexo de riscos, oportunidades e violência. Foi realizada uma pesquisa qualitativa com enfoque hermenêutico, usando 48 entrevistas em profundidade, analisadas com ferramentas teóricas em três estágios (descritivo, analítico e interpretativo). Os resultados mostram que a classificação dicotômica de jovens em resilientes versus não-resilientes (ou "com" versus "sem" problemas de comportamento) deixa de captar a maneira pela qual esses jovens vivenciam seu desenvolvimento, que é dinâmico e que muda de acordo com suas características, potencialidades, necessidades e interesses, além das oportunidades que encontram.

Desenvolvimento do Adolescente; Assunção de Riscos; Resiliência Psicológica; Violência
Recibido el 06/Jul/2014

Versión final presentada el 29/Oct/2015

Aprobado el 03/Nov/2015 